\title{
Basic Design Architecture of Congestion Notification System: A Real Time Road Traffic Information Enabler
}

\author{
J. A. Odeleye*, L. I. Umar \\ Nigerian Institute of Transport Technology (NITT) P.M.B 1148, Basawa Road Zaria, Kaduna State, NIGERIA.
}

\begin{abstract}
Road traffic congestion is a prominent challenge of today's urban center. As a push factor of urban centers, it impact negatively on socio-economic well-being of cities. However, contemporary innovative transport technology of Intelligent Transport System (ITS) is bridging the traveler information gaps, through installation and deployment of smart transport infrastructure such as Congestion Notification System at critical traffic intersections and points that aggravate road traffic congestion. This paper therefore provides a detailed explanation on the configuration and basic architecture of a primary Congestion Notification System (CNS) stating its working principles in providing real time road traffic congestion level information to motorist, prior entering the congestion zones or section of the road. Thus, engendering informed decision by motorists on alternative routes rather than the congested route.
\end{abstract}

Keywords: ITS, traffic congestion, inductive loops, CCTV, WiFi

\section{INTRODUCTION}

Traffic congestion on road networks is characterized by slower speeds, longer trip times, and increased vehicular queuing. Traffic congestion is one of the major challenges of contemporary urban areas. It is a price that motorist pay for the various benefits derived from urbanization and blooming economic activity. Because land is scarce and road capacity is expensive to construct, it would be uneconomical to invest in road expansion at a level that travel will ensure congestion-free. Vehicle volume is increasing exponentially with the increase in automobile dependency, while the road infrastructures are slowly improving and this leads to increase traffic congestion. Indeed, because demand for travel depends on the cost, improvements in travel conditions induce people to take more trips, and it would probably be impossible to eliminate congestion. However, the congestion level and its attendant effects can be reduced by introducing control mechanism to inform motorist of its occurrences prior to its real time occurrences so as to decide on diverting to other alternative routes, should there be one. Different technologies are there to detect traffic congestion and making congestion management more efficient. But these technologies have several drawbacks, such as installation, power and maintenance problems, complexity, cost, etc.

*Corresponding author (Tel: +234 (0)803 590 5619)

Email addresses: joshuaodeleye@gmail.com (J. A. Odeleye), IlawalO10@gmail.com (L. I. Umar)
Downs [1] road traffic congestion law, as quoted by Odeleye [2] which states "efforts to relieve automobile congestion through highway and other infrastructure expansion will simply be undone by continuous growth in the demand for travel; thus fulfilling the now well-known and documented "Law of Congestion". This is now widely known as generated or induced demand. Notwithstanding the reality of the Down's thesis, few transport policies ultimately reflect the reality of the law. Rather planners, politicians and citizens alike all hope that the next road expansion will bring the elusive solution to congestion woes [3]. Nevertheless, Downs [4] warns that "ring road and by passes may not provide a solution to peak-hour traffic loads: the most important thing to understand about peak-hour traffic congestion is that once it has appeared in a region, it cannot be eliminated or even substantially reduced". Lawrence [5] shares this view of maximizing the efficiency and capacity of existing transportation networks because of the continued increase in traffic volume and the limited construction of new highway facilities in urban, intercity, and rural areas. This is because as demand increases relative to the limited construction of new roads, there is tendency of recurring congestion especially in the industrialized world and as well as in developing nations. Therefore, building or improving highways to relieve congestion might be short-lived in areas where population is projected to grow substantially". Hence, technology enabled solutions such as CNS capable of providing motorists with real time information on road congestion levels, prior entering the congested road sector, as advocated by Odeleye [2], is found to be more cost-effective 




Figure 1: Block Diagram of the Design.

that investing in road capacity expansion and/or upgrade as the ultimate practical solution for relieving road traffic congestion in any urban setting.

The International Transport Forum [6] in line with the above submission highlighted the need and basis for promoting such system thus "The large diffusion of ubiquitous sensing devices, portable remote and edge computing capabilities, IT Infrastructures, new data treatment and analysis protocols, data-feed algorithms and wide spread, fast, reliable and robust communication networks all lead to an unprecedented revolution in the way in which transport stakeholders can optimized multiple and converging goals and outcomes". Similarly, Sakshi et al. [7] reveals the need for Congestion Notification System sighting the increase in the number of vehicles which led to traffic congestion as a problem. Hence suggested that, to overcome the problems, a system has to be designed which can alert for congestion which they named "Automatic Traffic congestion detection and alert system". Abdullah et al. [8] are with a similar view which is focused on reducing traveler time on roads (highways in particular) through the same system of traffic congestion notification system. Hence, stressed the need for road users to get timely information and thus be able to avoid congested path and look for alternative routes. Though, taking different approaches, all reviewed literatures reiterate the need for CNS. It is against this, the idea of resolving Congestions problems through the use of Technology (CNS) come forth.

Congestion notification system is not a new method of traffic smoothing and congestion alleviation. Many authors put forth effort to solve congestion problems by exploring different divergent technologies and methods to suit the situation at hand considering the geographical sphere and the nature of motorist driving in that particular sphere. The work of Sabya [9] uses image processing technology that sends information of congested area to the road side unit using Zigbee protocol. This signal is then send to traffic surveillance system which then activates a traffic light and congestion control system for day light sequences. The main set back of this project is its daylight only function. Another similar work that explore image processing is that of Pejman [10] which uses Image processing to automatically estimates traffic density then accommodate information regarding the size of traffic in highway and detects the occurrence of accidents and violations on highways. The is way too complicated to the type of system in Nigeria. The work of Vikramaditya et al. [11] is also based on Image Processing in coordination with Intelligent Traffic Controller System that is designed to favour emergency vehicles detection. Though the project is not meant for traffic congestion notification, its function can easily be extended to such by manipulating the detection algorithm and replacing the Radio frequency identification (RFID) with more flexible and broadband system. This is also a solution highlighted in the work of José et al. [12] that proposed a Collaborative and Opportunistic Traffic Monitoring System called COTraMS to monitor traffic using IEEE 802.11 networks.

However, the main factor to be considered in choosing any (CNS) is its adaptability to the area of function. The level of technology and the simplicity of maintenance and installation are also factors to be considered. This make this proposed design suitable for application in the Nigerian contex.

\section{DESIGN DESCRIPTION}

The design placed a Metallic Pole at the notification zones and at the congestion zones to houses the support and house the Display (Notification) units, as well as the power and communication units respectively. The Metallic Pole at the congestion zones housed the two CCTV camera mounted facing both sides of the roadway, and 


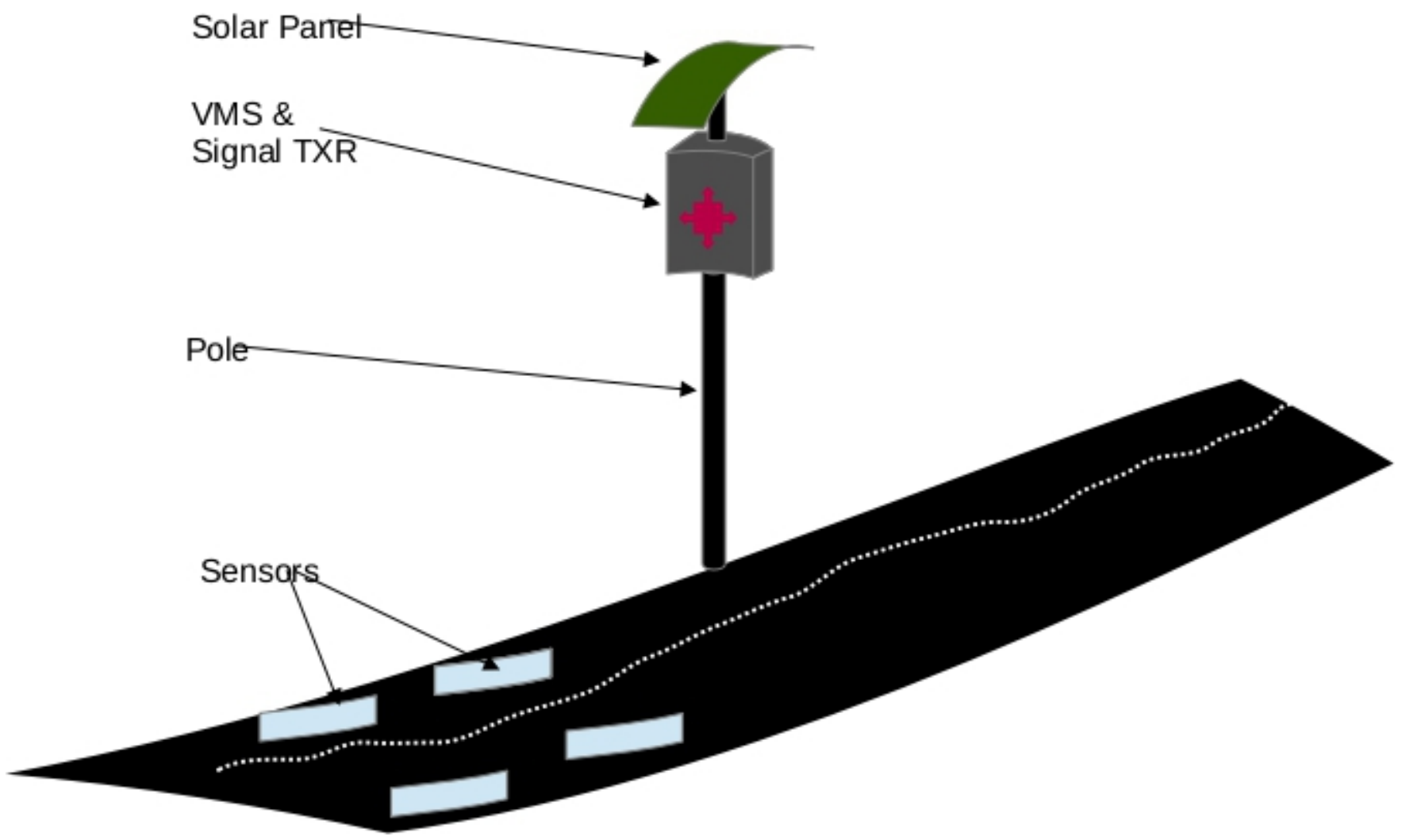

Figure 2: Data Acquisition and Sensor System (authors proposed arrangement).

a communication module for sending information to the control and monitoring center (Back-end Office). There are four inductive loops or optical sensors at each lane within this zones placed at the approaching side of the roadway. At the notification zones, the Metallic Pole housed the Variable message sign (VMS), a display unit for information dissemination. In this manner, travelers from/to approaching routes will have the congestion real-time information, prior entering the known congested zone. All the Metallic Pole has a solar powered system at its top to provide the power needed to drive the systems. A block diagram of the proposed CNC is shown in Fig. 1.

\section{HOW CNS WORKS}

Figure 2 shows the graphical illustration of Inductive loops or optical sensors spread along the approaching routes at an interval of three hundred (300) meters along each of the lane side (to and fro) to monitor the slowing down (rate of deceleration) of moving vehicles (very slow movement of vehicles means congestion is happening) at the congestion zones. This information would be sent to the back-end office via the selected communication link. Also, two CCTV cameras would be placed at the congestion zones to confirm the presence of congestion by sending Real-TimeInformation (RTI) each time deceleration occurs and in this design; images of the congestion zone at an interval of $20 \mathrm{sec}$ are sent to the back office. The choice of image instead of video is to lesser the data being transmitted. The CCTV will send sequence of images at an interval of 20 seconds to the backhouse when there is sudden slowdown of vehicle o the engaged road section as detected by the inductive loop sensor embedded in the pavement.

\subsection{Information Display}

Variable Massage Sign (VMS) boards placed at the notification zones by the lane sides (to and from) notify the presence of congestion ahead and indicates the opening of alternative route. The VMS gets this information from the back-end office via the selected communication links.

\subsection{Power Supply}

The power requirement of the system consist that of; the VMS (15W), Loops (160W, 40W each), CCTV (15W) and the communication modules (100W) which is $12 \mathrm{~V}$ DC voltage operated. This design considers the use of dual solar power system arrangements (500W) placed on the Metallic Polefor reliability and security respectively.

\subsection{Communication Link (Wireless)}

3G/GPRS/DSRC communication links are selected for this design because of their availability and reliability in Nigeria. These communication links were used to send information from the backend office to the VMS and from Communication module at the Metallic Pole(inductive loops information and CCTV real time image) to the backend office. 


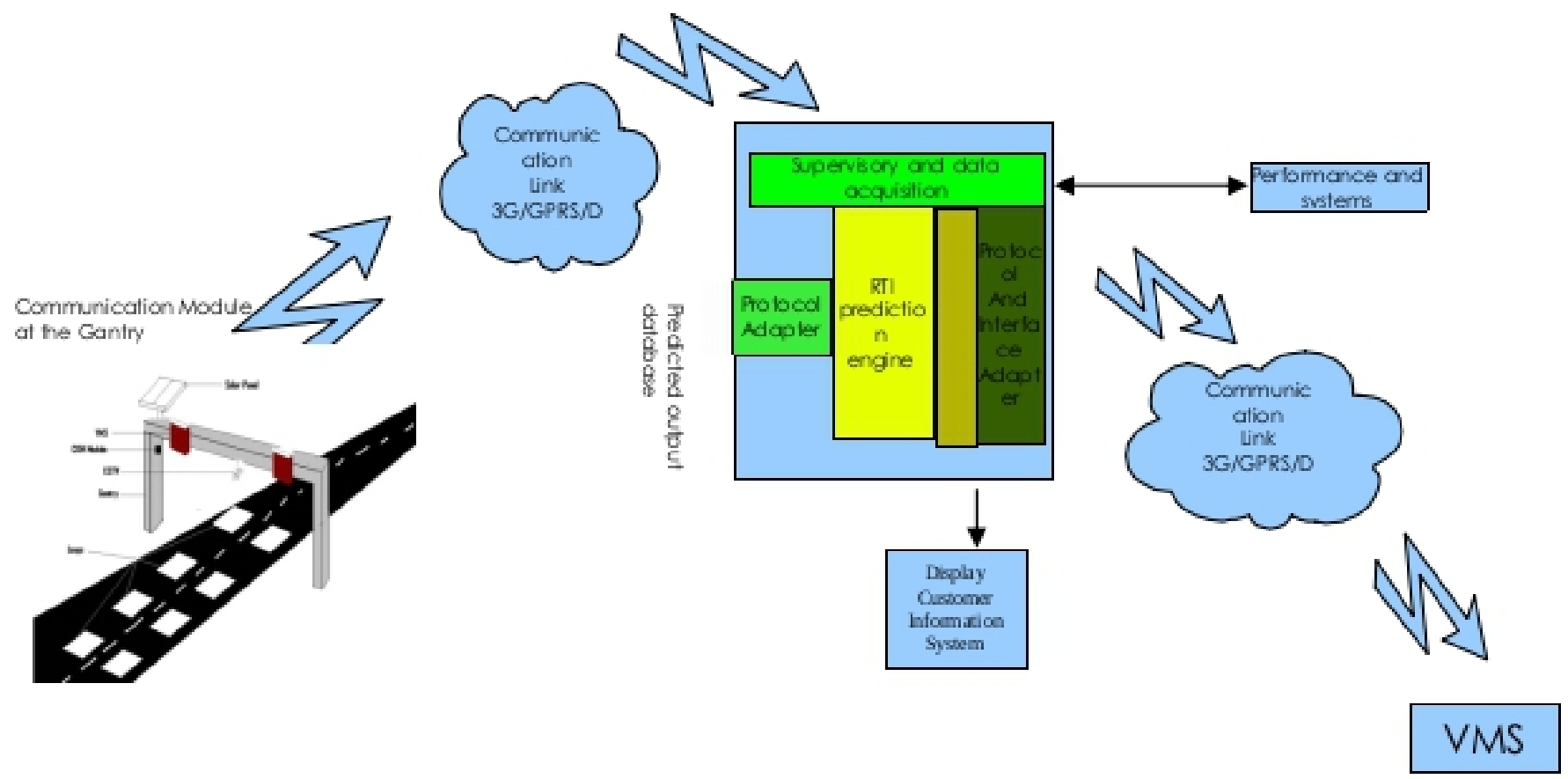

Figure 3: Architecture of the CNS (proposed by the authors).

\subsection{Back-end Office}

This was the control center housing the communication module to receive and transmit data and a display screen. The screen would be displaying the images of the congestion zones to confirm the presence of the congestion. In addition, it housed data storage facility to store the received data for future forecast and control module for activation of by-pass route opening sequence. Source of energy: Solar energy and PHCN.

\section{DESIGN ARCHITECTURE}

The Design architecture of this Congestion Notification system describes its major components, their relationships (structures), and how they interact with each other. Architecture serves as a blueprint for a system. It provides an abstraction to manage the system complexity and establish a communication and coordination mechanism among components and the subsystems. It also defines a envisaged structured solution that will meet all the technical and operational requirements, while optimizing the common quality attributes like performance and security. Figure 3 shows the design architecture of the intended congestion notification system, with details of technical specifications listed in Table 1.

\section{CONCLUSION}

This paper presents the basic design architecture of a primary road traffic congestion notification system based on CCTV and Inductive Loops sensing and image comparison, being a tested technological solution for easing congestion at critical zones of road, such as major urban intersections. At deployment of this soft, digital road systems infrastructure, a road traffic information enabler, that enables real-time traffic information for motorists to take informed travel decision of detouring into alternative routes to avoid congested corridor, engendered reduced transit time and energy saving amongst motorists. Also, road traffic congestion induced pollution as well as road crashes associated with waiting in line on congested corridor will be minimized reasonably at deployment of CNS on urban highway. Again, this application would help in holistic urban traffic management such that live sharing of road events/ incidents through videos and pictures at various locations on digital mobile map is made possible by its deployment of CNS at critical zones of urban highway.

Summarily, while it's ideal and good to expand and upgrade existing highway for free flow of traffic, it will however not be out of place, if cities authorities across Nigeria could consider reasonable investment in soft road infrastructure. Because, the embedded benefits of these infrastructure, aside from the primary role of congestion management, are enormous. For example, cities need data to determine the performance levels of their network, this soft infrastructure is capable of providing such data like traffic volumetric pattern, travel time at critical zones etc real time across urban network. This data when acquired real time engender better road traffic management plan as well as informed road investment decision over time and space.

\section{References}

[1] A. Downs, "Stuck in traffic. coping with peak-hour traffic congestion," Journal of Planning Education and Research, vol. 12, no. 3, pp. 257-259, 1992.

[2] J. A. Odeleye, "A study of road traffic congestion in selected corridors of metropolitan, lagos," Ph.D. dissertation, University of Lagos Nigeria, Lagos, 2008. 
Table 1: Component List.

\begin{tabular}{|c|c|c|c|c|c|c|}
\hline Component & Description & $\frac{\text { Quantity }}{16}$ & Voltage & $\begin{array}{c}\text { Power } \\
40 \mathrm{~W}\end{array}$ & Installation & Maintenance \\
\hline Inductive loop & & & & $40 \mathrm{~W}$ & $\begin{array}{l}\text { Buried along the } \\
\text { roadway }\end{array}$ & $\begin{array}{l}\text { Maintenance } \\
\text { free }\end{array}$ \\
\hline CCTV & $\begin{array}{l}300 \mathrm{Mpx} \text {, high resolution, } \\
\text { water proof, sun protected etc }\end{array}$ & 4 & $12 \mathrm{~V}$ & $15 \mathrm{~W}$ & $\begin{array}{l}\text { At the middle of } \\
\text { the gantry }\end{array}$ & Replaceable \\
\hline VMS & $\begin{array}{l}\text { High resolution LED, water } \\
\text { proof and sun protected }\end{array}$ & 8 & $12 \mathrm{~V}$ & $15 \mathrm{~W}$ & $\begin{array}{l}\text { At each end-side } \\
\text { of the gantry }\end{array}$ & Replaceable \\
\hline Gantry & $\begin{array}{l}\text { Light metal with good earth- } \\
\text { ing and lightning protection }\end{array}$ & 4 & & & $\begin{array}{l}\text { Mounted across } \\
\text { the roadway }\end{array}$ & $\begin{array}{l}\text { Maintenance } \\
\text { free }\end{array}$ \\
\hline $\begin{array}{l}\text { Com Link Mod- } \\
\text { ule }\end{array}$ & 3G/GPRS/DSRC & 5 & $12 \mathrm{~V}$ & $50 \mathrm{~W}$ & $\begin{array}{l}\text { Mounted on the } \\
\text { gantry }\end{array}$ & Replaceable \\
\hline Power supply & Solar powered & 4 & $12 \mathrm{~V} \mathrm{DC}$ & $500 \mathrm{~W}$ & On the gantry & Replaceable \\
\hline
\end{tabular}

[3] C. Zegras, "Clearing the sky in santiago," United $\mathrm{Na}$ tions Centre for Human Settlements (UNCHS) Journal, vol. 4 , no. 2, p. 24, 1998.

[4] A. Downs, "Why traffic congestion is here to stay and will get worse. access, fall, 25," Journal of University of California Transportation Centre, pp. 19-25, 2004.

[5] A. K. Lawrence, "Traffic Detector Handbook," Federal Highway Administration Turner-Fairbank Highway Research Center, 6300 Georgetown Pike, HRDO04, Room No. T204 McLean, 2008, vA 22101-2296. FHWA-HRT-06-108.

[6] International Transport Forum. (2019) ITF transport outlook 2019. [Online]. Available: www.itf-oecd.org

[7] S. Sakshi, K. K. Narendra, and M. Mukesh, "Automatic traffic technology," International Journal of Computing and Technology, vol. 1, no. 6, p. 296, 2014.

[8] A. Abdullah, A. Manzoor, and H. Ahmed, "An early alert system for traffic congestion based on social messages on smartphones," International Journal of Computer Applications, vol. 133, no. 11, pp. 27-29, 2016.

[9] S. K. Sabya, "Real-time traffic light-control and congestion avoidance system," International Journal of Engineering Research and Applications, vol. 2, no. 2, pp. 925-929, 2012.

[10] N. Pejman, "Automatic Traffic Estimation Using Image Processing," International Journal of Signal Processing, Image Processing and Pattern Recognition, vol. 5, no. 4, pp. 167-169, 2012.

[11] D. Vikramaditya, P. Amol, P. Kshitij, and S. S. Rathod, "Image processing based intelligent traffic controller," Undergraduate Academic Research Journal, vol. 1, no. 1, pp. 4-15, 2012.

[12] G. R. José, E. M. C. Miguel, H. Luís, and M. K. Costa, "COTraMS: A Collaborative and Opportunistic Traffic Monitoring System," Journal of Engineering Research and Applications, vol. 2, no. 2, pp. 926-929, 2012. 